\title{
Shenqi Lixin Decoction improves cardiac function in rats with adriamycin-induced heart failure through modulation of PGC-1a and mitochondrial apoptosis pathway
}

\author{
Jinlong Zhuang", Jian Zhu", Yan Dou, Xiaoqing Chen, Hua Chen, Xuean Liu, Genghai Lin, Fahui Ruan \\ Department of Cardiology, Xiamen University Affiliated Dongnan Hospital, Zhangzhou, China \\ Contributions: (I) Conception and design: G Lin, F Ruan, J Zhuang; (II) Administrative support: G Lin, F Ruan, J Zhuang; (III) Provision of study \\ materials or patients: J Zhuang, J Zhu, Y Dou; (IV) Collection and assembly of data: J Zhuang, J Zhu, X Chen, H Chen; (V) Data analysis and \\ interpretation: J Zhuang, J Zhu, X Liu; (VI) Manuscript writing: All authors; (VII) Final approval of the manuscript: All authors. \\ \#These authors contributed equally to this work. \\ Correspondence to: Genghai Lin; Fahui Ruan. Department of Cardiology, Xiamen University Affiliated Dongnan Hospital, Zhangzhou 363000 , China. \\ Email: lghai6868@163.com; afarun@163.com.
}

Background: Heart failure (HF) is a complex clinical syndrome and a serious manifestation or late stage of various heart diseases. This study aimed to explore the protective effects and underlying mechanisms of Shenqi Lixin Decoction (SQLXD) in HF.

Methods: A HF rat model was induced by intraperitoneal injection of adriamycin $(3 \mathrm{mg} / \mathrm{kg}$ in the first 3 weeks, $2 \mathrm{mg} / \mathrm{kg}$ in the next 3 weeks, once a week, subcutaneous injection, 6 weeks cumulative dose is $15 \mathrm{mg} / \mathrm{kg})$. After 4 weeks of intragastric administration of SQLXD $(9.975,19.95,39.90 \mathrm{~g} / \mathrm{kg}$, once a day, gavage), the indexes of cardiac function were measured by cardiac color Doppler ultrasound, the cardiac muscle structure and pathological changes were observed by transmission electron microscope, hematoxylineosin (HE) staining and Masson. The plasma N-terminal B-type natriuretic peptide (NT-proBNP) level and myocardial tissue adenosine triphosphate (ATP) content were detected by ELISA. FITC detected the cardiomyocyte apoptosis rate (CMAR) labeled Annexin V/PI. Expression of B cell lymphoma factor 2 (Bcl-2), Bcl-2 associated X (Bax), cysteine protease-3 (Caspase-3), and peroxisome proliferator-activated receptor $\gamma$ coactivator- $1 \alpha$ (PGC-1 $\alpha$ ) mRNA in myocardial tissue were detected by real-time PCR (RT-PCR). The expression of Bcl-2, Bax, Caspase-3 and P53 protein in myocardial tissue were detected by Western blot.

Results: Compared to the normal group, left ventricular end systolic diameter (LVSD), left ventricular end diastolic diameter (LVDD), CMAR and the expression of P53 protein, mRNA and protein of Bax and Caspase-3 were significantly increased in model group, while left ventricular ejection fraction (LVEF), left ventricular fractional shortening (LVFS), stroke volume (SV) and the expression of Bcl-2 protein, mRNA of PGC-1 $\alpha$ and Bcl-2 were significantly reduced. Compared to the model group, LVSD, LVDD, CMAR and the expressions of P53 protein, mRNA and protein of Bax and Caspase- 3 in the medium and high dose SQLXD groups and the control group were significantly decreased, while LVEF, LVFS, SV and the expression of Bcl-2 protein, mRNA of PGC-1 $\alpha$ and Bcl-2 were obviously increased. Pathological findings by transmission electron microscope, Masson, and HE staining all revealed protective effects of SQLXD on heart.

Conclusions: SQLXD can effectively protect HF rats' hearts. The potential mechanism may be related to the modulation of the expression of PGC-1 $\alpha$ and the mitochondrial apoptosis pathway.

Keywords: Shenqi Lixin Decoction (SQLXD); heart failure (HF); myocardial structure; cardiac function; cardiomyocyte apoptosis

Submitted Sep 18, 2021. Accepted for publication Oct 20, 2021.

doi: $10.21037 / \mathrm{atm}-21-5350$

View this article at: https://dx.doi.org/10.21037/atm-21-5350 


\section{Introduction}

Heart failure (HF) is a complex clinical syndrome in which impaired ventricular filling or pumping function is caused by an abnormality in heart structure or function. It is a serious manifestation or late stage of various heart diseases and a global-important public health issue (1). Current research (2) has shown that neuroendocrine-mediated myocardial remodeling is the main pathophysiological mechanism of the occurrence and development of HF, and cardiomyocyte apoptosis is its important pathological basis. In the past 20 years, although the treatment strategy for HF has gradually changed from "strengthening the heart, diuresis, and dilation of blood vessels" to improve the hemodynamic state in the past to the current longterm, a therapeutic strategy that mainly "inhibits abnormal neuroendocrine activation", however, the rate of rehospitalization and mortality of patients with $\mathrm{HF}$ are still high, and the prognosis is poor. Therefore, the treatment of $\mathrm{HF}$ at this stage still urgently needs to find new methods.

Traditional Chinese medicine (TCM) has a long history in the treatment of HF. Most of them are based on famous prescriptions from ancient books and are divided into various empirical prescriptions based on clinical experience. The clinical efficacy is good, but it has limitations and lacks modern evidence-based medical evidence and laboratory indicators. Nevertheless, due to the characteristics of multicomponent, multitarget, and multipathway drugs of Chinese medicine compound, it has an incomparable advantage over a single Western medicine in treating HF. TCM has been recommended for the management of HF by the Chinese Heart Failure Guidelines (1), and experts have developed a consensus of the diagnosis and treatment of chronic HF in a combination of TCM and Western medicine $(3,4)$. However, at present, the mechanism of treatment of HF by TCM is not yet fully understood. Most mechanism research improves clinical symptoms, hemodynamics, and neurohumoral regulation, and there is a lack of in-depth research on the pathophysiological mechanism of HF. Shenqi Lixin Decoction (SQLXD) is based on "Linggui Zhugan Decoction, Yangxin Decoction". SQLXD regimen consists of: ginseng $20 \mathrm{~g}$, astragalus $20 \mathrm{~g}$, cassia twig $10 \mathrm{~g}$, epimedium $20 \mathrm{~g}$, Lepidium seed $15 \mathrm{~g}$, salvia $15 \mathrm{~g}$, tuckahoe $20 \mathrm{~g}$, atractylodes $15 \mathrm{~g}$, agrimony $30 \mathrm{~g}$, motherwort $15 \mathrm{~g}$ and licorice $10 \mathrm{~g}$. Its preliminary clinical and basic research has shown that it can improve the clinical symptoms of patients with HF and increase activity tolerance and improve heart function indexes $(5,6)$. However, it improved the hemodynamics of rats with HF caused by doxorubicin and reduced cytoinflammatory factors $(7,8)$, but the mechanism of its intervention in HF is currently unclear. Previous studies on the effects of SQLXD on cardiac function mainly focused on hemodynamics, cardiac ejection function, BNP, cytoinflammatory factors, myocardial metabolism indicators, and some apoptotic genes. This study aimed to explore the effect of SQLXD on HF from the pathophysiological mechanism for the first time, and further explored the mechanism of SQLXD in the treatment of HF from the molecular level. We present the following article in accordance with the ARRIVE reporting checklist (available at https://dx.doi.org/10.21037/atm-215350).

\section{Methods}

\section{Animals}

One hundred and five healthy Wistar male rats, 6-7 weeks old, SPF grade, body weight $(200 \pm 20)$ g, purchased from Shanghai Slack Laboratory Animal Co., Ltd., certificate number: SCXK (Shanghai) 2017-0005 were used for this study. They were raised in separate cages in the Animal Experimental Center of Southeast Hospital Affiliated with Xiamen University, free water, and food, 12 hours of light, temperature $(22 \pm 3){ }^{\circ} \mathrm{C}$, humidity $(50 \pm 8) \%$. Experiments were performed under a project license (No. 909DWLL201901) granted by ethics committee of Xiamen University Affiliated Dongnan Hospital, in compliance with the National Laboratory Animal Management Regulations and guidelines for the care and use of animals. A protocol was prepared before the study without registration.

\section{Agents}

SQLXD consisted of ginseng $20 \mathrm{~g}$, astragalus $20 \mathrm{~g}$, cassia twig $10 \mathrm{~g}$, epimedium $20 \mathrm{~g}$, Lapidium seed $15 \mathrm{~g}$, salvia $15 \mathrm{~g}$, tuckahoe $20 \mathrm{~g}$, atractylodes $15 \mathrm{~g}$, agrimony $30 \mathrm{~g}$, motherwort $15 \mathrm{~g}$ and licorice $10 \mathrm{~g}$. It was purchased from the outpatient Chinese pharmacy of Southeast Hospital Affiliated with Xiamen University. Enalapril maleate tablets (10 mg/tablet, Yangtze River Pharmaceutical Group Jiangsu Pharmaceutical Co., Ltd., Nanjing, China); doxorubicin hydrochloride for injection $(10 \mathrm{mg} /$ piece, Zhejiang Hisun Pharmaceutical Co., Ltd., Taizhou, China); hematoxylineosin (HE) staining solution (Beijing Yili Fine Chemicals 
Co., Ltd., Beijing, China); N-terminal B-type natriuretic peptide (NT-proBNP) and adenosine triphosphate (ATP) ELISA kit (Wuhan Fine Biotech Co., Ltd., Wuhan, China); Annexin V-FITC and PI kits, Trizol kits, two-step real-time PCR (RT-PCR) kits, lysates, BCA protein concentration determination kits, SDS-PAGE, Super-GL ECL ultrasensitive luminescent fluid (Shanghai Novland BioPharma Co., Ltd., Shanghai, China). Shanghai Integrated Biotech Solutions Co., Ltd. (Shanghai, China) synthesized PCR primers. Other materials included PVDF transfer membrane (Millipore, USA); horseradish peroxidase (HRP) labeled goat anti-rabbit IgG antibody (Shanghai Beyotime Biotechnology Co., Ltd., Shanghai, China); recombinant anti-Bcl-2 associated $\mathrm{X}$ (anti-Bax) antibody (E63), anti-B cell lymphoma factor 2 (anti-Bcl-2) antibody, recombinant anti- $\beta$-actin antibody (EPR16769), anti-cysteine protease-3 (anti-Caspase-3) antibody (EPR18297), anti-P53 antibody (PAb 240) (Abeam, USA).

\section{Apparatus}

IE3 3 Cardiac Color Doppler Ultrasound System (Philips, Netherlands); ST5020 Multifunctional Staining and Covering Machine, RM2245 Microtome (Leica Microsystems Co., Ltd., Germany); DW4-B Biological Microscope (Shanghai Leica Microsystems Trading Co., Ltd., Shanghai, China); JEM-1220 transmission electron microscope (Hitachi, Japan); BD-FACSCALIBUR flow cytometer (BD Bioscience, USA); MX3000P fluorescent quantitative PCR (qPCR) instrument (Agilent, USA); PowerPacTMHC electrophoresis instrument, 170-3940 semi-dry transfer Tank, 170-3932 transfer membrane filter paper, $\mathrm{XR}+$ gel imaging analysis system (Bio-Rad, USA); VE-180 vertical electrophoresis tank (China Tanon Technology Co., Ltd., Huzhou, China); Gel-Pro analyzer analysis software (Media Cybernetics, USA).

\section{Preparation of SQLXD}

Ginseng, Astragalus, Epimedium, Cassia twig, Lepidium seed, Salvia, Poria, Atractylodes, Agrimony, Leonurus, and Licorice were soaked in distilled water for 1 hour, decocted for 1 hour, then filtered with gauze. The filter residue was decocted with distilled water for $1 \mathrm{~h}$, filtered again, collected the filtrates, and then concentrated into the liquid with crude drug concentration of $0.998,1.995,3.990 \mathrm{~g} / \mathrm{mL}$, sterilized in aliquots, and stored in a refrigerator at $4{ }^{\circ} \mathrm{C}$ spares. The dose of SQLXD is calculated based on the body weight and surface area of adults $(60 \mathrm{~kg})$ and rats $(200 \mathrm{~g})$, and the conversion factor for humans and rats is 0.018 .

\section{HF model}

According to previous studies $(9,10)$, after feeding the rats for 7 days, 15 rats were selected as the normal group using the random number table method. The remaining rats were injected intraperitoneally with doxorubicin solution $(0.8 \mathrm{mg} / \mathrm{mL})$ at $3 \mathrm{mg} / \mathrm{kg}$ for the first 3 weeks and $2 \mathrm{mg} / \mathrm{kg}$ for the next 3 weeks. The normal group was injected intraperitoneally with the same amount of $0.9 \%$ saline once a week for 6 weeks; after 6 weeks, the rat heart was detected by color Doppler ultrasound. Left ventricular ejection fraction (LVEF) $<45 \%$ means that the HF rat model is successfully established. According to previous research $(7,8), 80$ successfully modeled HF rats were randomly divided into five groups, namely the model group and the control (enalapril) group $(2.1 \mathrm{mg} / \mathrm{kg})$ group, SQLXD low $(9.975 \mathrm{~g} / \mathrm{kg})$, medium $(19.95 \mathrm{~g} / \mathrm{kg})$ and high dose $(39.90 \mathrm{~g} / \mathrm{kg})$ groups with 16 rats in each group. The control and model groups were administered an equal volume of distilled water, 1 time/d, for 4 consecutive weeks. In the experiment, carefully observe and record the mental state, diet, activity, urine output, feces, hair, and other general conditions and death of the rats every day.

\section{Samples collection and storage}

After 4 weeks of treatment, rats fasted without water for 12 hours, hair removed, weighted, and intraperitoneally injected with $20 \%$ urethane $(1.5 \mathrm{~g} / \mathrm{kg})$ for anesthesia. Color Doppler ultrasound detected the heart function of rats. Blood $(5 \mathrm{~mL})$ was collected from the abdominal aorta and injected into EDTA anticoagulation tube, stand for $1 \mathrm{~h}$, centrifuge at $3,000 \mathrm{r} / \mathrm{min}$ for $10 \mathrm{~min}$, collect the plasma, store it in the refrigerator at $-20^{\circ} \mathrm{C}$, and use it for the detection of NT-proBNP level. Rats were sacrificed immediately after blood collection, the thoracic cavity was cut open, and the heart was quickly removed. After washing the blood with ice saltwater, the pericardium, blood vessels, and atrial tissues were cut off on ice. The left ventricular myocardium was collected and quickly cut approximately $3 \mathrm{~mm}^{3}$ of left ventricular apex myocardial tissue to detect myocardial cell apoptosis and take 3 copies of $1 \mathrm{~mm}^{3}$ myocardial tissue for HE stains, Masson stains and electron microscopic observation, respectively. The remaining left ventricular myocardial tissue was quickly 
fixed in liquid nitrogen after 10 minutes, transferred to a $-80{ }^{\circ} \mathrm{C}$ refrigerator for storage for quantitative RT-PCR (qRT-PCR), Western blot, and ATP content detection.

\section{Data collection}

\section{Heart function}

After 4 weeks of drug intervention, these rats were injected intraperitoneally with $20 \%$ urethane for anesthesia, fixed on the back, and depilated on the chest. The left ventricular end-systolic diameter (LVSD), left ventricular end-diastolic diameter (LVDD), LVEF, left ventricular fractional shortening (LVFS) and stroke volume (SV) are continuously measured for 3 cardiac cycles with a cardiac color Doppler ultrasound system (10 MHz probes).

\section{Pathological study}

The left ventricular myocardium sample was fixed in 4\% paraformaldehyde solution, dehydrated by gradient ethanol, transparent with xylene, and embedded in paraffin to make $4 \mu \mathrm{m}$ sections. Take 4 sections from each specimen and stain with HE, mount the slides with neutral gum, observe the pathological changes of rat myocardial tissue under an optical microscope and take pictures. Take 5 slices from each specimen, stain with Masson staining, mount the slides with neutral gum, observe the fibrosis degree of rat myocardial tissue under an optical microscope and take pictures. The left ventricular myocardium samples were fixed in $2.5 \%$ glutaraldehyde solution, rinsed 3 times with cold phosphate buffer, fixed with 1\% osmic acid, dehydrated with gradient ethanol, embedded with epoxy resin, and ultra-thin section. After that, 5 slices of each specimen were taken, and the ultrastructure of rat cardiomyocytes was observed under a lens electron microscope and photographed.

\section{Cardiomyocyte apoptosis rate (CMAR)}

Left ventricular myocardial tissue was washed with cold phosphate buffer and a single-cell suspension was prepared was prepared by the mechanical method. Put the myocardial tissue on a 200-mesh stainless steel net, place a petri dish underneath, first cut the tissue into $1 \mathrm{~mm}^{3}$ size with ophthalmic scissors, gently scrub the tissue, wash with cold PBS solution while rubbing, and then collect the cell suspension through a 300-mesh strainer. Centrifuge the cell suspension at 2,000 r/min for $5 \mathrm{~min}$, discard the supernatant, collect the cells, resuspend in $4^{\circ} \mathrm{C}$ precooled PBS solution, at 2,000 r/min, centrifuge for $5 \mathrm{~min}$, wash the cells twice, and adjust the cell density to $1 \times 10^{5}-$ $5 \times 10^{5} \mathrm{pcs} / \mathrm{mL}$. After aspirating and discarding the PBS, resuspend the cells in $300 \mu \mathrm{L}$ of Binding Buffer, add $5 \mu \mathrm{L}$ of Annexin V-FITC, mix gently, avoid light, and incubate at room temperature for $15 \mathrm{~min} ; 5 \mathrm{~min}$ before using the machine, add $5 \mu \mathrm{L}$ of PI for staining. Add $200 \mu \mathrm{L}$ of the binding buffer before detection. The BD-FACSCALIBUR flow cytometer was used to detect the apoptosis rate of rat cardiomyocytes.

\section{NT-proBNP and ATP detection}

Pretreated plasma was taken to detect the level of NTproBNP in rat plasma according to the ELISA kit instructions. Left ventricular myocardial tissue samples were precooled, lysed, homogenized and centrifuged to extract tissue protein, prepare tissue homogenate and detect the ATP content of rat myocardial tissue according to the instructions of the ELISA kit.

qRT-PCR detect Bcl-2, Bax, Caspase-3, and peroxisome proliferator-activated receptor $\gamma$ coactivator-1 $\alpha$ (PGC1a) mRNA expression level

Left ventricular myocardium tissue samples were treated with the Trizol method to extract total RNA. Follow the instructions of the two-step real-time fluorescent quantitative polymerase chain reaction kit and reverse transcription into cDNA. The primers were designed and synthesized by Shanghai Integrated Biotech Solutions Co., Ltd. Take $2 \mu \mathrm{L}$ of cDNA in a $30 \mu \mathrm{L}$ system according to the operation steps of the above kit instructions, and use the MX3000P fluorescent qPCR instrument to analyze Bcl-2, Bax, Caspase-3, and PGC-1 $\alpha$ for amplification, PCR reaction conditions: $94{ }^{\circ} \mathrm{C}$ pre-denaturation for $3 \mathrm{~min}$, 1 cycle; $94{ }^{\circ} \mathrm{C}$ denaturation for $15 \mathrm{~s}, 55^{\circ} \mathrm{C}$ annealing for $40 \mathrm{~s}, 72{ }^{\circ} \mathrm{C}$ extension for $50 \mathrm{~s}, 40$ cycles. Using $\beta$-actin as an internal reference, the $2^{-\Delta \Delta \mathrm{Ct}}$ method was used to detect the relative expression of Bcl-2, Bax, Caspase-3 and PGC-1 $\alpha$ mRNA were analyzed. The experiment was repeated three times. See Table 1 for primer sequences.

\section{Western blot detect proteins expression levels}

Left ventricular myocardial tissue samples were added with $500 \mu \mathrm{L}$ of precooled lysate, ground, homogenized, lysed on ice and centrifuged to extract tissue protein. Use the BCA method to determine the protein level quantitatively. SDS-PAGE electrophoresis was conducted with $50 \mu \mathrm{g}$ as the loading amount per well, electrotransferred to PVDF membrane, adding $5 \%$ skimmed milk powder solution, 
Table 1 Primer sequence of PCR

\begin{tabular}{lll}
\hline Gene & Primer sequence $\left(5^{\prime}-3^{\prime}\right)$ & bp \\
\hline Bcl-2 & Forward: CGACTTTGCAGAGATGTCCA & 223 \\
& Backward: ATGCCGGTTCAGGTACTCAG & \\
Bax & Forward: TGCAGAGGATGATTGCTGAC & 207 \\
& Backward: GGAGGAAGTCCAGTGTCCAG & \\
Caspase-3 & Forward: ACGGACCTGTGGACCTGA & 151 \\
& Backward: GGCGCAAAGTGACTGGAT & \\
PGC-1 $\alpha$ & Forward: GGGCACATCTGTTCTTCC & 197 \\
& Backward: TCCCGTAGTTCACTGGTC & \\
$\beta$-actin & Forward: CGTAAAGACCTCTATGCCAACA & 131 \\
& Backward: GGAGGAGCAATGATCTTGATCT & \\
\hline
\end{tabular}

$\mathrm{Bcl}-2$, B cell lymphoma factor 2; $\mathrm{Bax}, \mathrm{Bcl}-2$ associated $\mathrm{X}$; Caspase-3, cysteine protease-3; PGC-1 $\alpha$, peroxisome proliferator-activated receptor $\gamma$ coactivator-1 $\alpha$.

blocking at $37^{\circ} \mathrm{C}$ for 1 hour, adding diluted recombinant anti-Bcl-2 recombinant anti-Bax, anti-Caspase- 3 and anti-P5 3 antibody $(1: 2,000)$, recombinant anti- $\beta$-actin antibody $(1: 10,000)$, incubated overnight at $4^{\circ} \mathrm{C}$, washed 4 times with TBST, 10 min each time. Add diluted HRPlabeled goat anti-rabbit IgG antibody $(1: 2,000)$, incubate at $37^{\circ} \mathrm{C}$ for $2 \mathrm{~h}$, wash 4 times with TBST, 10 min each time. After treatment with a supersensitive luminescent liquid Super-GL ECL, it was exposed, developed, and fixed in a dark room. After taking pictures with the American Bio-Rad gel imaging analysis system, $\beta$-actin was used as an internal reference. Analyze with Gel-Pro Analyzer software and calculate the ratio of the gray value of Bcl-2, Bax, Caspase-3 and P53 to the gray value of $\beta$-actin as relative levels of protein expression.

\section{Statistical analysis}

The experimental data were statistically analyzed using SPSS 22.0 software, all data are shown as the mean \pm standard deviation (SD). The least significant difference (LSD) $t$-test is used to compare the two groups that meet the normal distribution and homogeneity of variance, and the rank-sum test is used for those that do not meet the normal distribution and homogeneity of variance. Oneway analysis of variance (ANOVA) was used for comparison between multiple groups. $\mathrm{P}<0.05$ indicates that the difference is statistically significant.

\section{Results}

\section{Effects of SQLXD on heart function}

Compared with the normal group, the LVSD and LVDD of the model group were significantly increased $(\mathrm{P}<0.01)$, and the LVEF, LVFS, and SV were significantly decreased $(\mathrm{P}<0.01)$; compared with the model group, LVSD and LVDD of rats in the medium and high dose SQLXD groups and control group were significantly reduced $(\mathrm{P}<0.01)$, and LVEF, LVFS, and SV were significantly increased $(\mathrm{P}<0.01)$. See Figure 1.

\section{Effects of SQLXD on pathological changes of cardiac tissue}

HE stains showed that the structure of the myocardial cells in the normal group were clear, neatly organized, compact, without degeneration, necrosis, no edema and congestion in the interstitium, and no inflammatory cell infiltration. In the model group, myocardial cells were arranged disorderly, severely swollen, degenerated, and necrotic, muscle fibers were swollen and broken, and the interstitium was obviously edema, accompanied by inflammatory cell infiltration. In the low dose SQLXD group, myocardial cells were arranged disorderly and loosely, partly swollen, and necrotic, muscle fibers were swollen, interstitial edema, and a little infiltration of inflammatory cells. In the medium dose SQLXD group, myocardial cells were slightly disordered, without obvious edema, the muscle fibers were disordered, the structure was unclear, and the interstitium was mild edema. In the control group and the high dose SQLXD groups, the myocardial cells were evenly organized, the nucleus was enlarged and partially lost, the muscle fibers were slightly swollen, no breaks and the interstitium was slightly edema. See Figure 2.

\section{Effects of SQLXD on myocardial fibrosis}

Masson staining showed that the myocardial tissue fibers of the normal group were arranged neatly and tightly, with only a small amount of cord-like blue collagen fibers; the model group rats' myocardial tissue fibers were arranged disorderly and loosely, with a large number of sheet-like blue collagen fibers proliferating. The degree of myocardial tissue fibrosis in the low dose SQLXD group was less than that of the model group, but a large number of small pieces of blue collagen fiber hyperplasia were still seen. The degree of myocardial tissue fibrosis was significantly reduced in the control group, and the medium and high dose SQLXD 

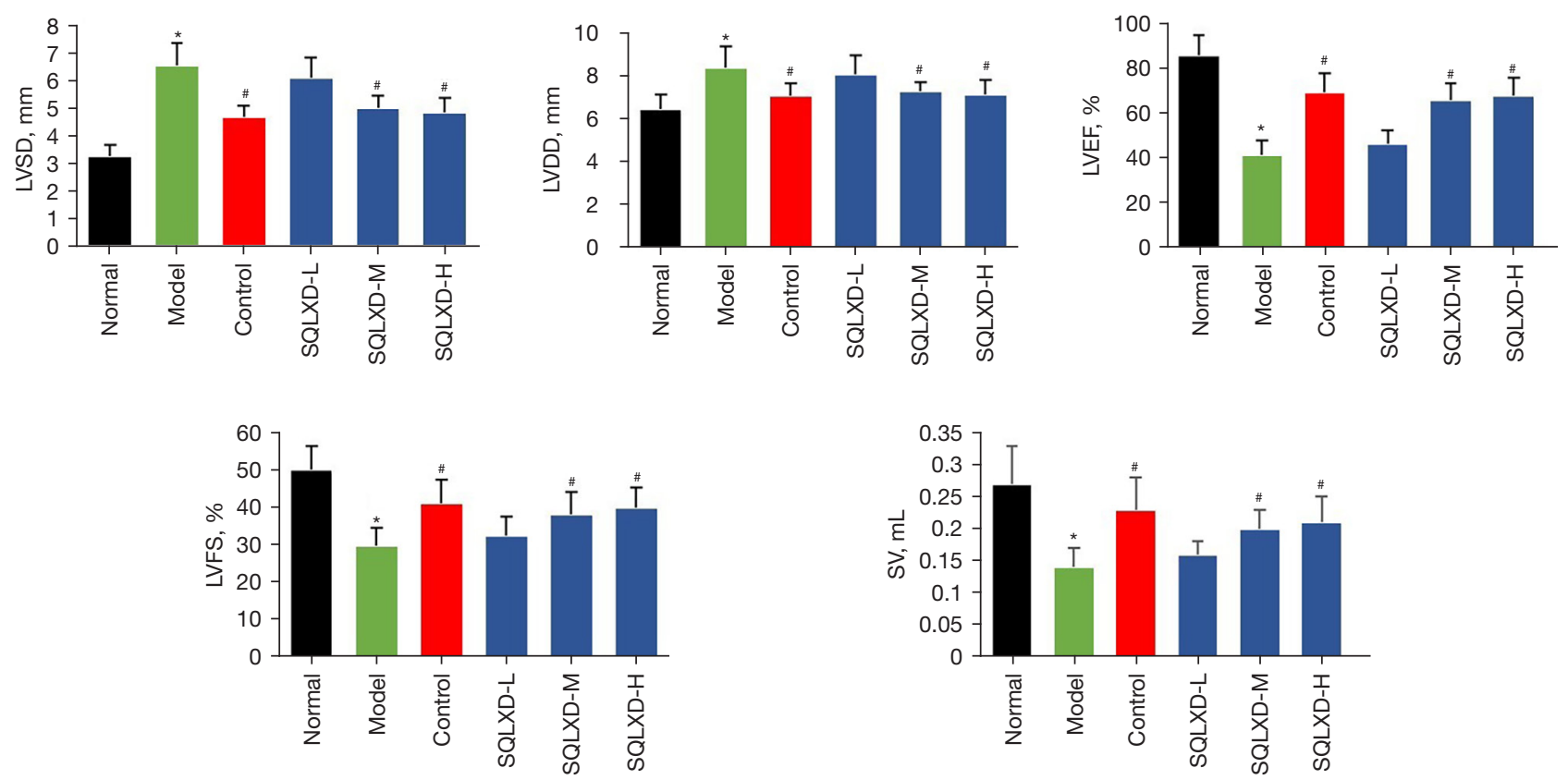

Figure 1 Effect of SQLXD on cardiac function in rats with $\mathrm{HF}(\bar{x} \pm s, \mathrm{n}=13) .{ }^{*} \mathrm{P}<0.01$ vs. normal group; ${ }^{*} \mathrm{P}<0.01$ vs. model group. SQLXD, Shenqi Lixin Decoction; HF, heart failure; L, low dose; M, medium dose; H, high dose; LVSD, left ventricular end-systolic diameter; LVDD, left ventricular end-diastolic diameter; LVEF, left ventricular ejection fraction; LVFS, left ventricular fractional shortening; SV, stroke volume.

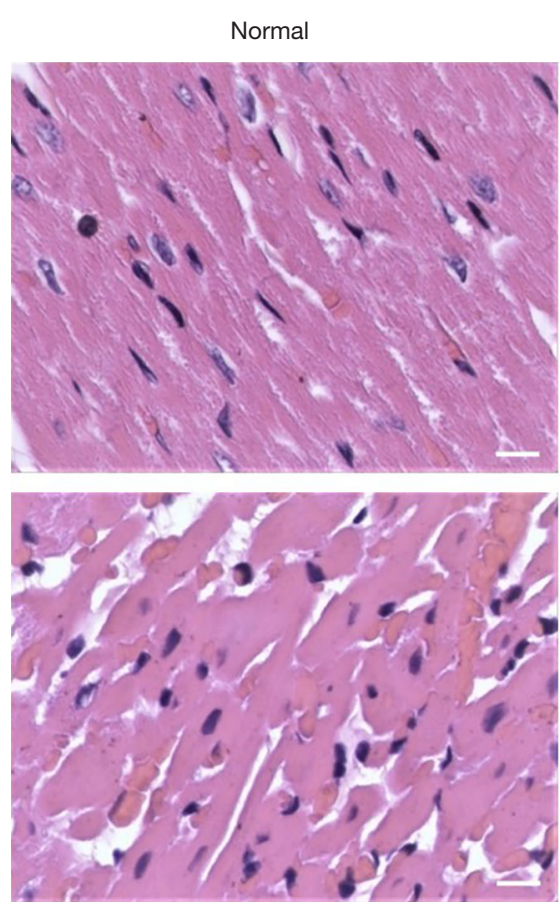

SQLXD-L
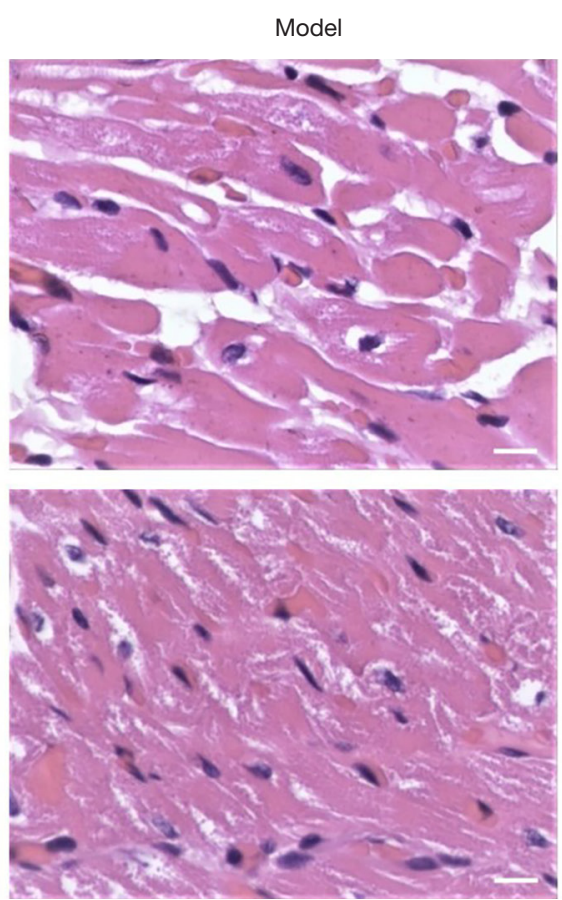

SQLXD-M
Control
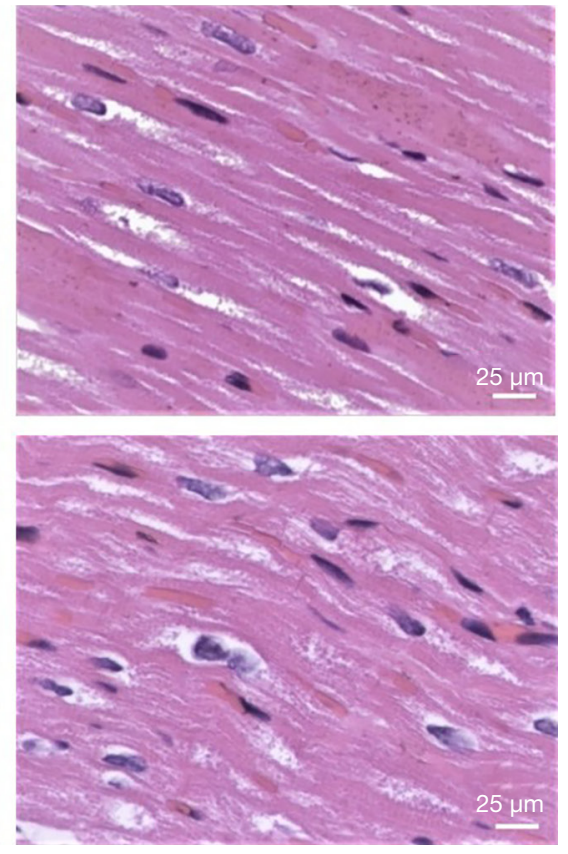

SQLXD-H

Figure 2 Pathological morphology changes in myocardial tissue of rats in each group (HE stains, $\times 400)$. HE, hematoxylin-eosin; SQLXD, Shenqi Lixin Decoction; L, low dose; M, medium dose; H, high dose. 

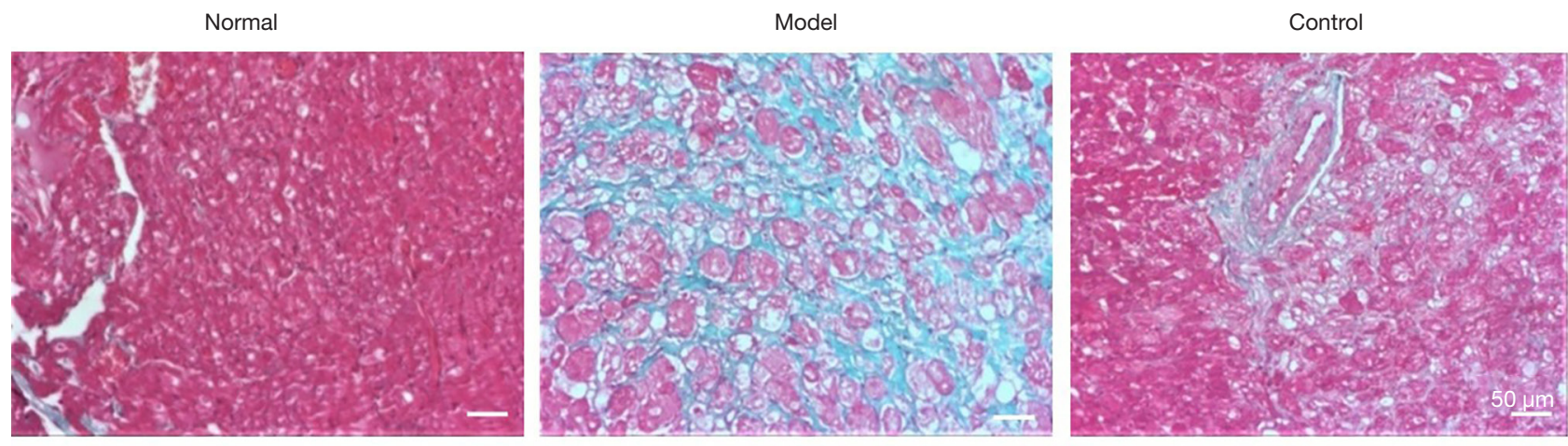

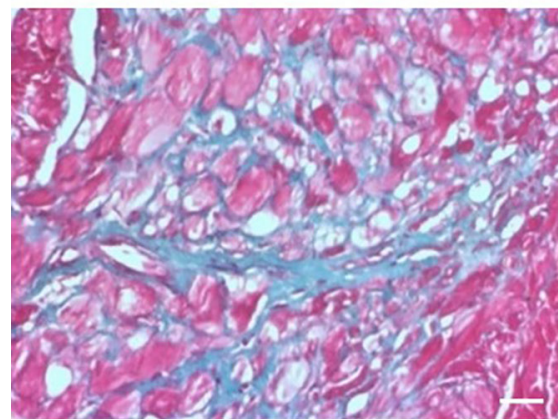

SQLXD-L

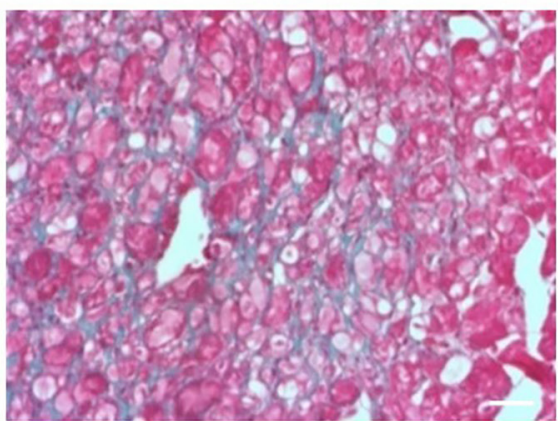

SQLXD-M

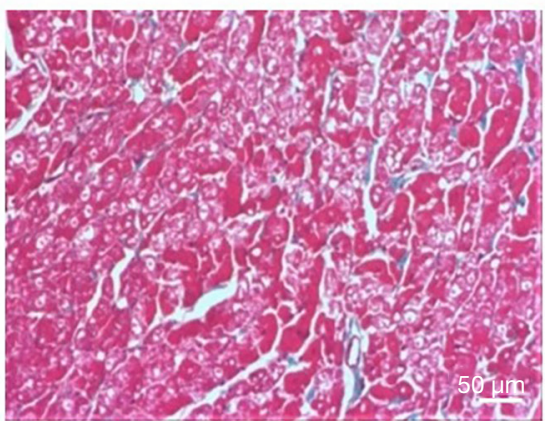

SQLXD-H

Figure 3 Pathological morphology changes in myocardial fibers of rats in each group (Masson staining, ×200). SQLXD, Shenqi Lixin Decoction; L, low dose; M, medium dose; H, high dose.

groups were compared to the model group and scattered small focal or strip-shaped blue collagen fibrils could be seen. See Figure 3.

\section{Effects of SQLXD on super-microstructure of myocardial cells}

Observed under a transmission electron microscope, the mitochondria of the myocardial cells in the normal group have different sizes, but the structure is complete without swelling, the inner cristae are clear, the muscle fibers are neatly organized and the light and dark bands are clear. The mitochondria of the myocardial cells in the model group were incomplete, swollen, dissolved, visible vacuolation, internal cristae dissolution and rupture, muscle fiber edema, severe dissolution. In the low dose SQLXD group, the mitochondria of myocardial cells were partially swelled, dissolved, vacuole-like degeneration, internal cristae dissolution, and partial muscle fiber dissolution. In the medium dose SQLXD group, the mitochondria of myocardial cells are disordered, partially swollen, rarely dissolved, no vacuoles are seen, a small part of the internal cristae is dissolved, and the muscle fibers are partially dissolved, which is less than the model group. In the high dose SQLXD group and control group, the mitochondria of myocardial cells were partially swollen, but the structure was still intact, and there was no dissolution, and most of the internal ridges were clear and less soluble. The muscle fibers were less soluble compared with the model group. See Figure 4.

\section{Effects of SQLXD on CMAR}

Compared with the normal group, the CMAR of the model group was significantly increased $(\mathrm{P}<0.01)$. Compared with the model group, the CMAR of the medium and high dose SQLXD groups and control group were significantly decreased $(\mathrm{P}<0.01)$. See Figure 5 .

\section{Effects of SQLXD on plasma level of NT-proBNP and myocardial tissue level of ATP}

Compared with the normal group, the plasma NT-proBNP level and the myocardial tissue ATP level of the model group were significantly increased $(\mathrm{P}<0.01)$. Compared with the model group, the plasma NT-proBNP level and 

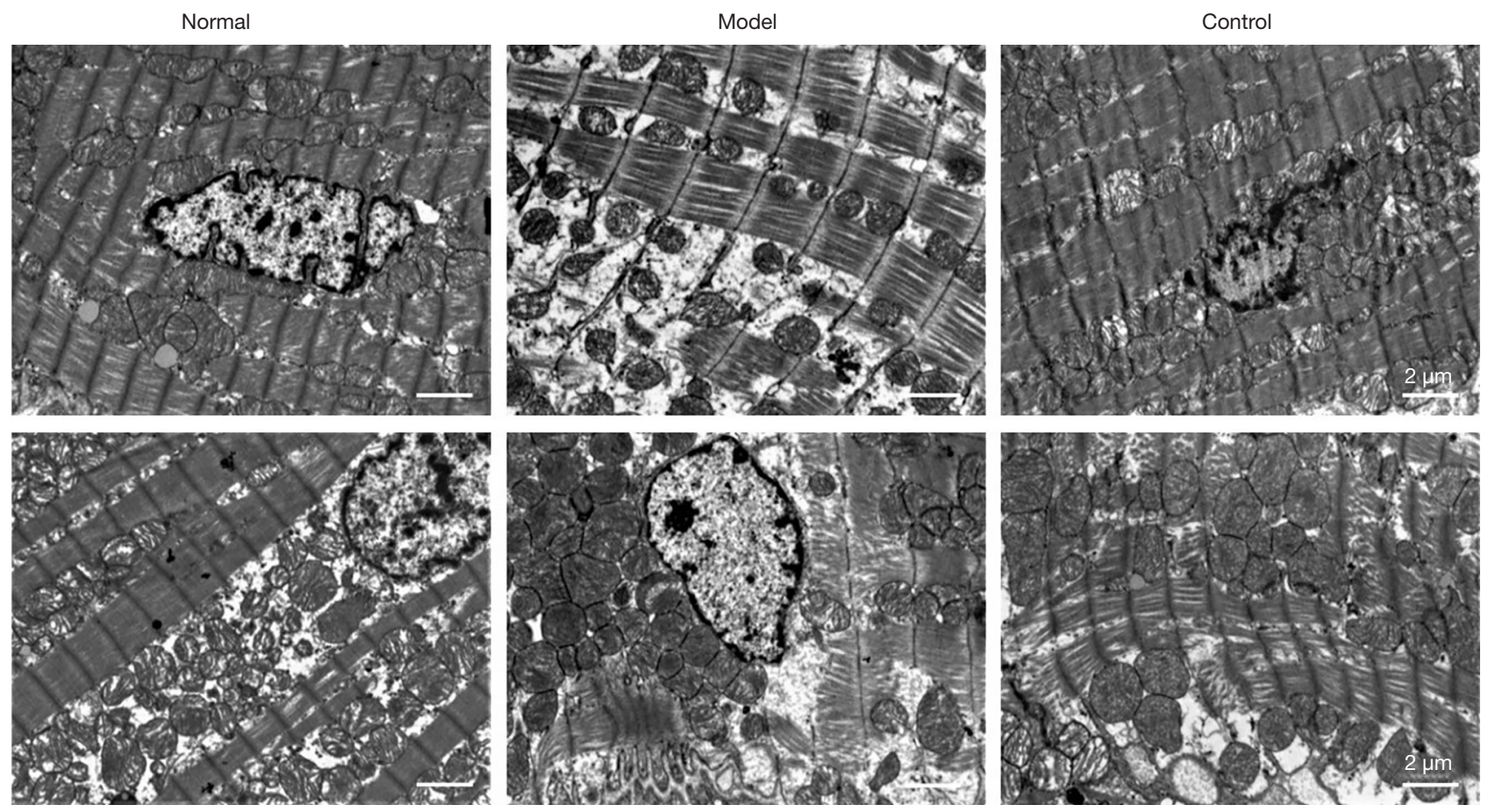

SQLXD-M

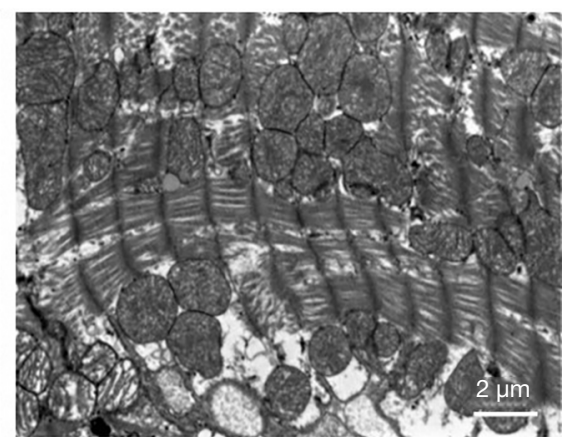

SQLXD-H

Figure 4 Ultrastructural changes in the myocardial cell of rats in each group (transmission electron microscope, $\times 5,000$ ). SQLXD, Shenqi Lixin Decoction; L, low dose; M, medium dose; H, high dose.

myocardial tissue ATP level in the medium and high dose SQLXD groups and control group were significantly reduced $(\mathrm{P}<0.01)$. See Figure 6 .

\section{Effects of SQLXD on Bcl-2, Bax, Caspase-3 and PGC-1a $m R N A$ expression levels}

Compared with the normal group, the expression of Bcl-2 and PGC- $1 \alpha$ mRNA in the model group were significantly reduced $(\mathrm{P}<0.01)$, and the expression of Bax and Caspase- 3 mRNA were significantly increased $(\mathrm{P}<0.01)$. Compared with the model group, Bcl-2 and PGC- $1 \alpha$ mRNA expression in the medium, high dose SQLXD groups and control group were significantly increased $(\mathrm{P}<0.01)$. In contrast, Bax and Caspase- 3 mRNA expression were significantly decreased $(\mathrm{P}<0.01)$. See Figure 7.

\section{Effects of SQLXD on Bcl-2, Bax, Caspase-3, and P53 protein expression}

Compared with the normal group, the expression of Bcl-2 protein in the model group was significantly reduced $(\mathrm{P}<0.01)$, and the expression of Bax, Caspase- 3 and P53 protein were significantly increased $(\mathrm{P}<0.01)$. Compared with the model group, the expression of Bcl-2 protein in the medium, high dose SQLXD groups and control group were significantly increased $(\mathrm{P}<0.01)$, and the expression of $\mathrm{Bax}$, Caspase- 3 , and $\mathrm{P} 53$ proteins were significantly decreased $(\mathrm{P}<0.05,0.01)$. See Figure 8 .

\section{Discussion}

The occurrence and development mechanism of HF has always been a research focus on the cardiovascular field. The current research believes that HF is a chronic and spontaneously progressive disease. The abnormal activation of the neuroendocrine system leads to myocardial remodeling, which is the main pathology of the occurrence and development of HF. The cardiomyocyte apoptosis is its important pathological basis, is the main reason for the loss of cardiomyocytes, and plays an important role in the pathogenesis of HF (2). Therefore, the effective intervention of cardiomyocyte apoptosis can reduce myocardial remodeling and delay the progression of HF, thereby improving the pump function of HF (11). The preliminary clinical and animal experiment research 

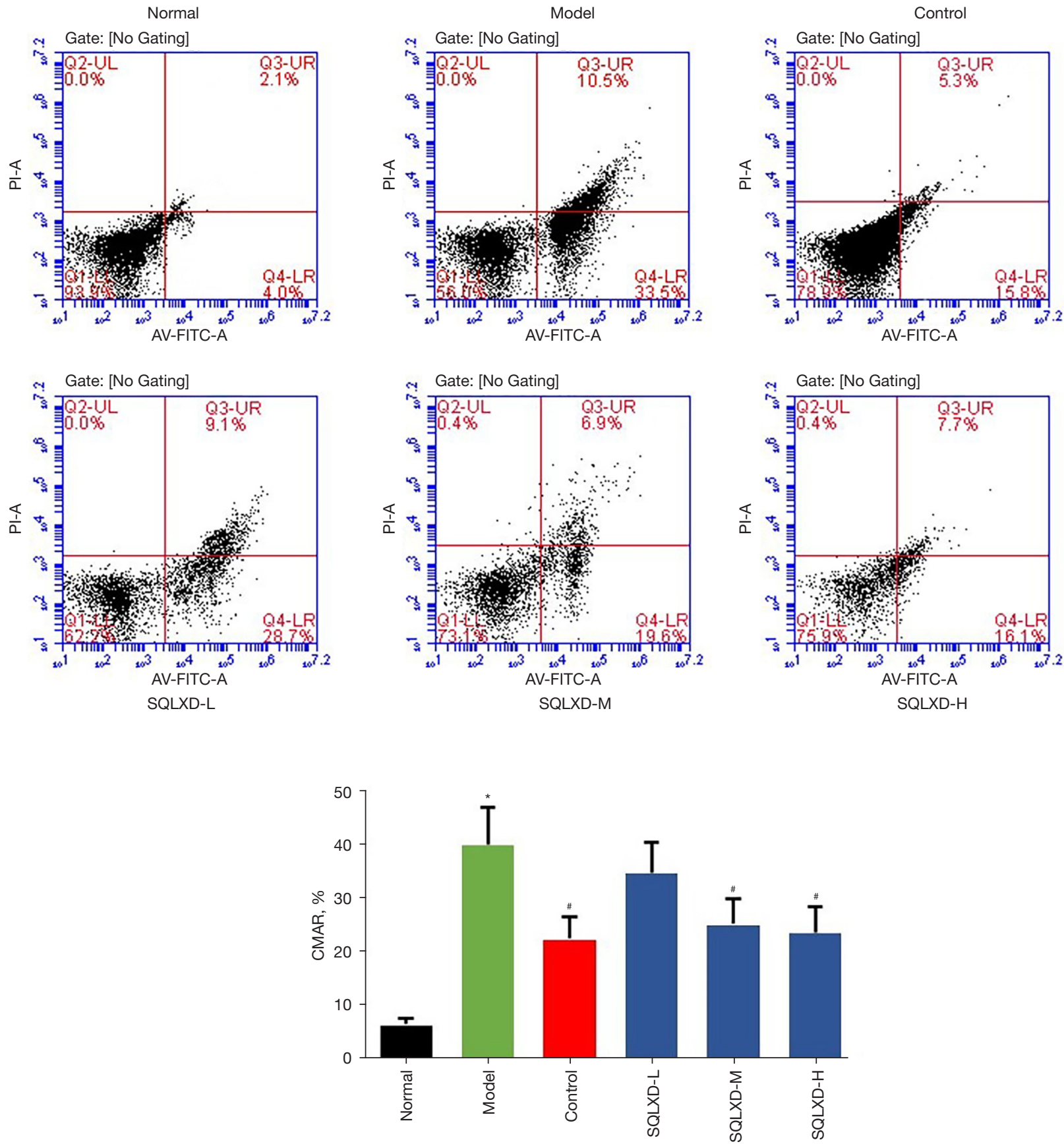

Figure 5 Effect of SQLXD on CMAR in rats with $\mathrm{HF}\left(\bar{x}_{ \pm}, \mathrm{n}=13\right)$. ${ }^{*} \mathrm{P}<0.01$ vs. normal group; ${ }^{\#} \mathrm{P}<0.01$ vs. model group. SQLXD, Shenqi Lixin Decoction; CMAR, cardiomyocyte apoptosis rate; HF, heart failure; L, low dose; M, medium dose; H, high dose.

has proved that SQLXD is an effective prescription for improving heart function and hemodynamics $(5-8)$. To investigate further the effect of SQLXD on improving heart function and myocardium, this study used echocardiography to detect cardiac function. NT-proBNP, CMAR, and related apoptosis genes and protein expressions of rats in each group were also detected. This study found that compared with the normal group, the CMAR and NTproBNP of the model group were significantly increased, and the cardiac function indexes (LVEF, LVFS, SV) were 

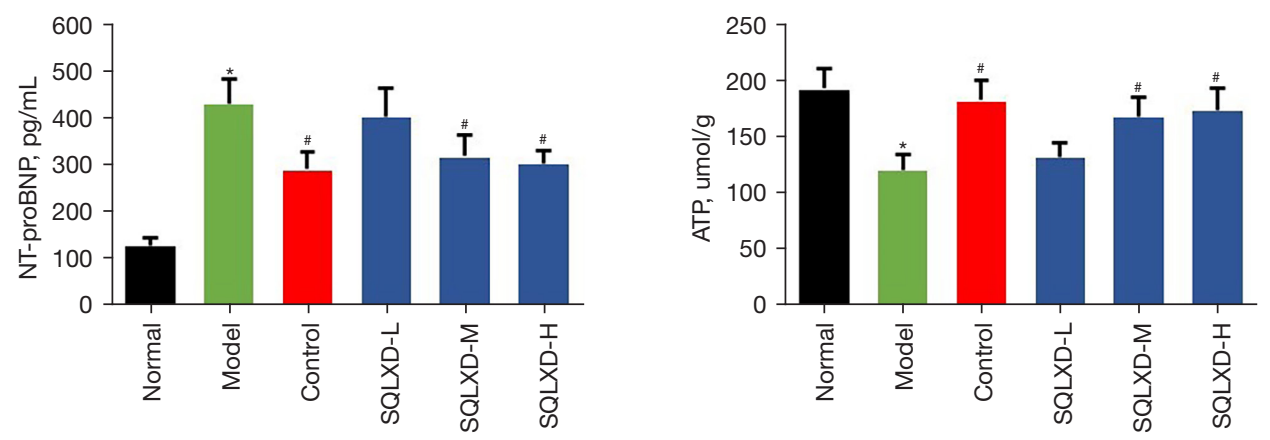

Figure 6 Effect of SQLXD on plasma NT-proBNP level and ATP content in the myocardium of rats with $\mathrm{HF}(\bar{x} \pm s, \mathrm{n}=13)$. ${ }^{*} \mathrm{P}<0.01 v s$. normal group; ${ }^{\text {P }}<0.01$ vs. model group. SQLXD, Shenqi Lixin Decoction; NT-proBNP, N-terminal B-type natriuretic peptide; ATP, adenosine triphosphate; HF, heart failure; L, low dose; M, medium dose; H, high dose.
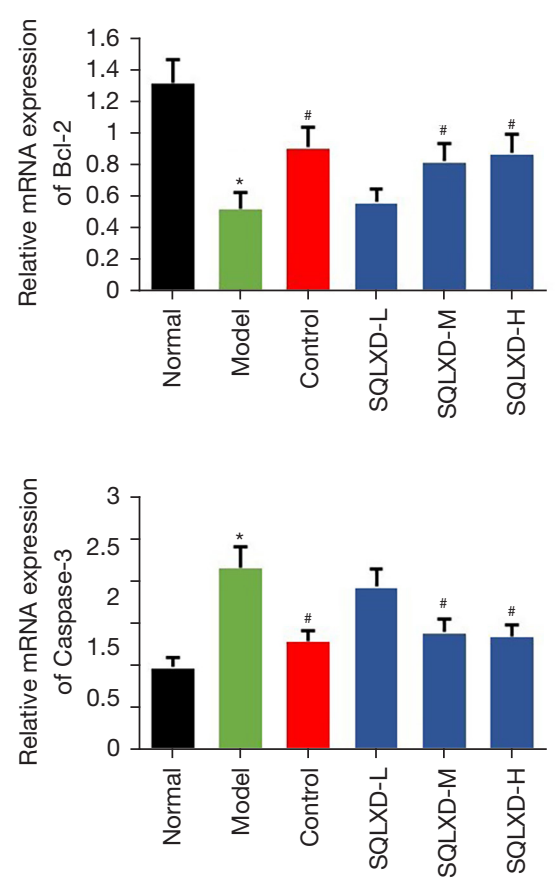
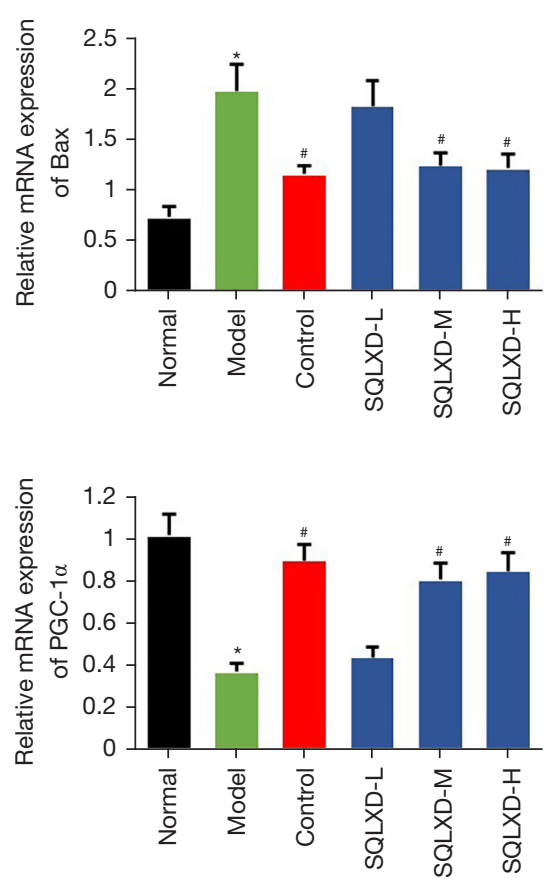

Figure 7 Effect of SQLXD on the expression of Bcl-2, Bax, Caspase-3 and PGC-1 $\alpha$ mRNA in myocardial tissues of rats with HF ( $\bar{x} \pm s$, $\mathrm{n}=6)$. ${ }^{*} \mathrm{P}<0.01$ vs. normal group; ${ }^{*} \mathrm{P}<0.01$ vs. model group. SQLXD, Shenqi Lixin Decoction; Bcl-2, B cell lymphoma factor 2 ; Bax, Bcl-2 associated X; Caspase-3, cysteine protease-3; PGC-1 $\alpha$, peroxisome proliferator-activated receptor $\gamma$ coactivator- $1 \alpha$; HF, heart failure; L, low dose; $\mathrm{M}$, medium dose; $\mathrm{H}$, high dose.

significantly decreased, indicating that there was severe cardiomyocyte apoptosis in the process of HF causing a large number of loss of cardiomyocytes, leading to myocardial contractile dysfunction, which in turn causes a significant decrease in heart function. This result is consistent with the pathophysiological changes of HF. After the intervention of SQLXD, the CMAR and NT-proBNP of rats in each experimental group decreased compared with the model group, and the cardiac function indexes (LVEF, LVFS, SV) also increased. The high dose group changed significantly, indicating that SQLXD can effectively inhibit cardiomyocyte apoptosis and improve heart function in rats with HF. There is a dose-dependent relationship, and the middle and high doses have significant effects. This study 

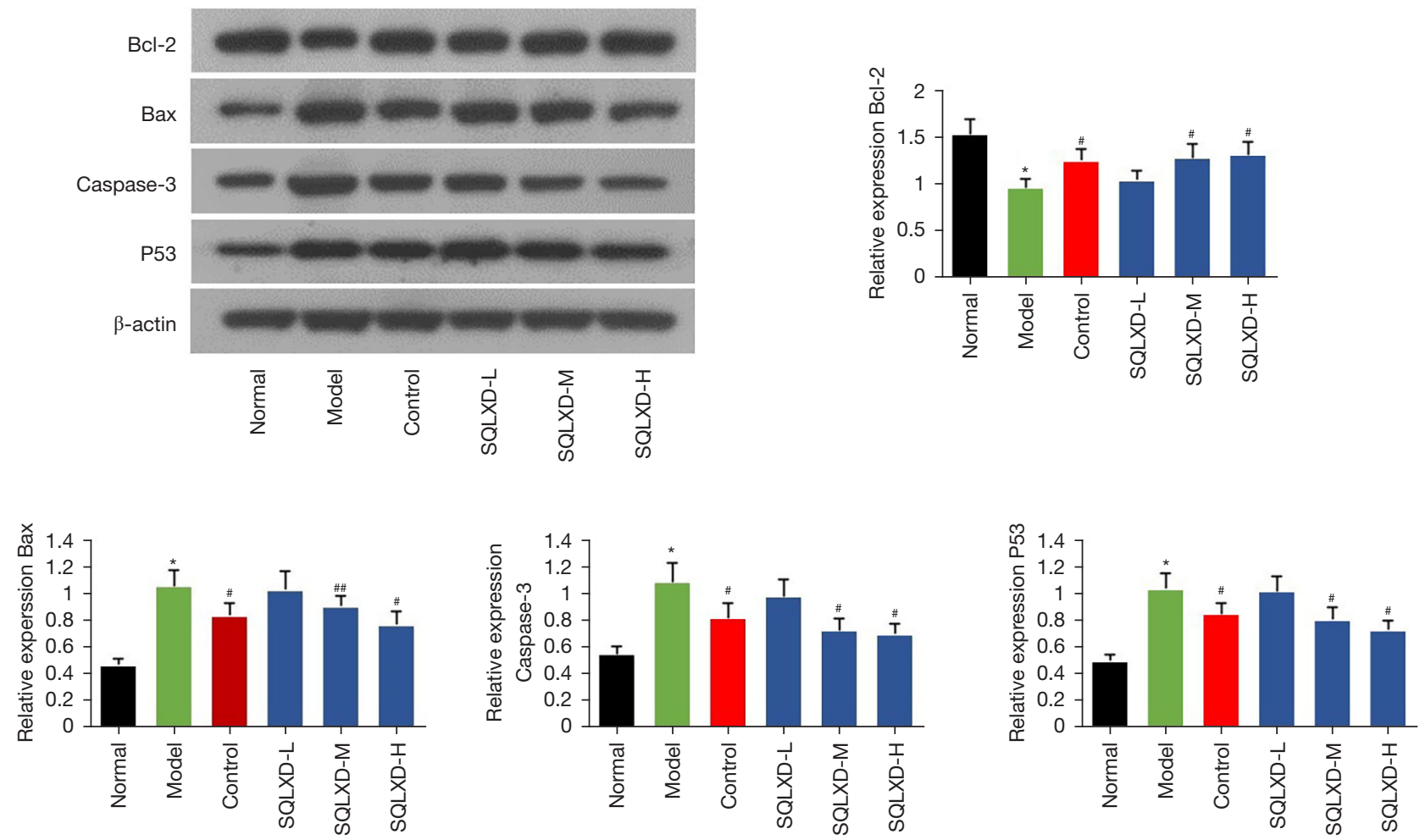

Figure 8 Effect of SQLXD on the expression of Bcl-2, Bax, Caspase-3 and P53 protein in myocardial tissues of rats with HF $(\bar{x} \pm s, \mathrm{n}=6)$. ${ }^{*} \mathrm{P}<0.01$ vs. normal group; ${ }^{\mathrm{P}}<0.01,{ }^{\#} \mathrm{P}<0.05$ vs. model group. SQLXD, Shenqi Lixin Decoction; Bcl-2, B cell lymphoma factor 2; Bax, Bcl-2 associated X; Caspase-3, cysteine protease-3; PGC-1 $\alpha$, peroxisome proliferator-activated receptor $\gamma$ coactivator-1 $\alpha$; HF, heart failure; L, low dose; $M$, medium dose; $\mathrm{H}$, high dose.

showed that SQLXD can effectively inhibit myocardial cell apoptosis, reduce myocardial cell loss, reverse myocardial remodeling, and improve cardiac function. This is the same as the results of related studies (11). At the same time, this study also found that enalapril is one of the most commonly used ACEI drugs in clinical practice, can significantly reduce cardiomyocyte apoptosis and improve cardiac function in rats with HF, which is consistent with relevant literature reports (12). There was no significant difference in rats' CMAR, NT-proBNP, and heart function indexes in the middle and high doses of SQLXD compared with the control (enalapril) group, indicating that the middle and high doses of SQLXD inhibited cardiomyocyte apoptosis and improvement of heart function is equivalent to that of enalapril.

The key research content of this experiment is how SQLXD inhibits myocardial cell apoptosis and improves heart function. Modern studies have shown that the heart is an important human organ with high energy consumption and high metabolism. It needs much energy to maintain its normal physiological functions and metabolic needs. Mitochondria are the main organelles for human energy metabolism. The heart is rich in mitochondria, which account for about $40 \%$ to $60 \%$ of the volume of cardiomyocytes. ATP is the most direct energy source required for various life activities of cells. Sixty percent to $80 \%$ of the energy in the normal adult heart comes from ATP produced by the mitochondrial fatty acid $\beta$ oxidation of cardiomyocytes. Studies have shown that the mitochondrial fatty acid $\beta$ oxidation ability in failing hearts is significantly reduced. The ATP content of the heart of patients with HF is about $30 \%$ to $40 \%$ lower than that of healthy people (13). This study found that compared with the control group, the ATP level of myocardial tissue in the model group was significantly reduced.

In contrast, the ATP level of myocardial tissue in each experimental group increased after the intervention of SQLXD. The middle and high dose groups increased significantly. PGC- $1 \alpha$ is a key regulator that promotes mitochondrial biosynthesis, enhances respiratory function 
and energy metabolism, and plays an important role in various energy metabolism processes of the body (14). PGC- $1 \alpha$ participates in regulating the physiological functions of myocardial mitochondria by regulating the coupled respiration of myocardial mitochondria, promoting the production of myocardial ATP, and then regulating the contractile function of the heart (15). The expression of PGC- $1 \alpha$ in myocardial tissue is abundant. PGC- $1 \alpha$ mRNA is overexpressed in the myocardial tissue of newborns, accompanied by the activation of mitochondria and the metabolic conversion from glycolysis to $\beta$-oxidation of fatty acids (16), similarly, the lack of PGC-1 $\alpha$ in myocardial tissue can lead to mitochondrial biosynthesis and energy metabolism disorders, leading to decreased heart function, $\mathrm{HF}$, and sudden death (17). This study also found that compared with the normal group, the PGC- $1 \alpha$ mRNA in the myocardium of the model group was significantly reduced.

In contrast, the PGC- $1 \alpha$ mRNA in the myocardium of the rats were significantly increased after the intervention of the medium and high doses of SQLXD. This study showed that the expression of PGC-1 $\alpha$ in the myocardial tissue is significantly decreased during $\mathrm{HF}$, resulting in a significant decrease in ATP content, which in turn leads to mitochondrial energy metabolism disorders, and it is difficult to maintain the energy requirements for normal myocardial activities. This result is the same as the above-mentioned related studies $(13,17)$, and SQLXD can significantly enhance the expression of PGC$1 \alpha$ in the myocardial tissue of rats with $\mathrm{HF}$, increase the content of ATP, thereby improve the energy metabolism of myocardial mitochondria, reduce cell apoptosis, and improve heart function. The effects mentioned above of SQLXD are inseparable from its royal drugs ginseng and astragalus. Modern pharmacological studies have shown that ginseng and astragalus are rich in ginsenoside $\mathrm{Rg} 1$, astragalus polysaccharides, etc., respectively, and ginsenoside Rg1 can up-regulate the expression of PGC$1 \alpha$ in myocardial cells, increases myocardial tissue ATP content, improve myocardial energy metabolism (18). Astragalus polysaccharides can regulate the expression of myocardial tissue PGC- $1 \alpha$, increase the ratio of ATP/ADP, ATP/AMP, increase energy supply, and reduce myocardial apoptosis (19).

In addition to effectively improving myocardial energy metabolism and reducing cell apoptosis, whether SQLXD mediates the expression of related genes and proteins in the myocardial cell mitochondrial apoptosis pathway is another focus research of this experiment. Cardiomyocyte apoptosis is an important pathological link in the process of $\mathrm{HF}$, a key turning point for $\mathrm{HF}$ from compensation to decompensation, and is regulated by a variety of apoptosisrelated genes. Myocardial mitochondria are not only factories for biosynthesis and energy metabolism but also the regulatory center of cardiomyocyte apoptosis, which is mainly regulated by the Bcl-2 protein family, including anti-apoptotic proteins (such as Bcl-2, Bcl-XL, etc.) and pro-apoptotic proteins (such as Bax, Bad, etc.), among which Bcl-2 and Bax are the most important members of the $\mathrm{Bcl}-2$ protein family. The ratio of Bcl-2/Bax determines the survival or death of cells $(20,21)$. Studies have shown that the anti-apoptotic protein $\mathrm{Bcl}-2$ resides in the outer mitochondrial membrane. Its role is to maintain the integrity of mitochondria, control the opening of mPTP, and inhibit the release of cytochrome C; the pro-apoptotic protein Bax resides in the cytoplasm. It reduces the mitochondrial membrane potential and causes the permeability of the outer membrane to increase, leading to the release of a large amount of cytochrome $\mathrm{C}$ and part of the apoptotic precursor AIF into the cytoplasm. And then, cytochrome $\mathrm{C}$ activates Apaf-1 and forms apoptotic bodies with Caspase-9, further activating Caspase-3 and Caspase-7, thereby inducing cell apoptosis (22). This study found that compared with the normal group, the expression of Bcl-2 mRNA and protein in the myocardial tissue of the model group were significantly reduced, the expression of Bax and Caspase-3 mRNA and protein were significantly increased, and the myocardial CMAR was also significantly increased. The expression of $\mathrm{Bcl}-2 \mathrm{mRNA}$ and protein in myocardial tissue of the rats were significantly increased after the intervention of middle and high doses of SQLXD, the expression of Bax and Caspase- 3 mRNA and protein were significantly decreased. The myocardial CMAR was also significantly decreased. P53 gene is an important gene in the apoptosis family. It is a transcription factor that promotes apoptosis. Its expression product is called P53 protein, a sequence-specific DNA binding protein that regulates transcription. It participates in the process of cardiomyocyte apoptosis and accelerates HF's progress (23). After DNA damage in cardiomyocytes, activated P53 protein induces Bax transcription, up-regulates Bax protein expression, and induces Noxa and Puma transcription simultaneously. The two competitively bind to Bcl-2 and down-regulate the expression of Bcl-2 (24). It leads to a decrease in the ratio of $\mathrm{Bcl}-2 / \mathrm{Bax}$, which initiates the mitochondrial apoptotic pathway to induce cardiomyocyte 
apoptosis and accelerate the progression of HF (25). Studies have found that the use of P53 inhibitors can reduce $\mathrm{H}_{2} \mathrm{O}_{2}$-induced cardiomyocyte apoptosis, thereby improving heart function (26). In addition, P53 protein also destroys the ability of myocardial damaged mitochondria to degrade autophagy, leading to myocardial mitochondrial dysfunction and promoting the development of HF (27). This study found that compared with the normal group, the expression of P53 in the myocardial tissue of the model group was significantly increased, the expression of Bax and Caspase-3 were also significantly increased, the expression of Bcl-2 was significantly decreased, and the myocardial CMAR was also significantly increased. Indicating that P53 mediates the mitochondrial apoptotic pathway and promotes cardiomyocyte apoptosis. This result is consistent with other studies $(24,25)$. Compared with the model group, the expression of P53 in myocardial tissue of rats were significantly reduced after the intervention of medium and high doses of SQLXD, the expression of Bax and Caspase-3 were also significantly reduced. However, the expression of Bcl-2 was significantly increased. At the same time, myocardial CMAR was significantly reduced. This study shows that cardiomyocyte apoptosis is mediated by the mitochondrial apoptosis pathway in the process of HF. SQLXD can regulate the expression of cardiomyocyte apoptosis-related genes in both directions, and it can up-regulate the anti-apoptotic gene Bcl-2. It can also down-regulate the expression of pro-apoptotic genes Bax, Caspase-3, and P53, intervene in cardiomyocyte apoptosis from the mitochondrial apoptosis pathway, reduce myocardial cell loss, thereby reverse myocardial remodeling, and improve cardiac function. This may be the reason One of the molecular mechanisms of improving HF. The effects mentioned above of SQLXD may be related to the main drugs contained in the prescription, such as ginseng, astragalus, salvia miltiorrhiza, epimedium, etc. Modern pharmacological studies have shown that the ingredients of the prescription mainly contain ginsenosides, astragaloside IV, tanshinone acid, epimedium, etc. Among these, ginsenosides can inhibit the expression of Caspase-3 protein in cardiomyocytes of $\mathrm{HF}$ rats, reduce cell apoptosis and reduce serum BNP levels, thereby improving heart function (28). Astragaloside IV can protect mitochondrial function and reduce the release of cytochrome $\mathrm{C}$, blocks the mitochondrial apoptotic pathway, and inhibits cardiomyocyte apoptosis $(29,30)$. Tanshinone IIA can inhibit the expression of Bax and P53 proteins in cardiomyocytes, thereby reducing cardiomyocyte apoptosis (31). Epimedium Glycoside can down-regulate $\mathrm{P} 53$ and Caspase- 3 protein expression in myocardial tissue, thereby inhibiting myocardial cell apoptosis (32).

In summary, SQLXD can improve the myocardial structure, myocardial tissue energy metabolism, and cardiac function in rats with $\mathrm{HF}$ and reduce myocardial cell apoptosis. The mechanism of action may be related to the regulation of myocardial PGC- $1 \alpha$ and mitochondrial apoptosis pathways. Whether SQLXD can effectively intervene in the process of myocardial fibrosis in rats with HF is the next direction for the research team to study further. Under the guidance of TCM theory, this study uses modern scientific experimental methods to reveal that SQLXD improves HF from the pathophysiological mechanism, provides objective experimental evidence for the prevention and treatment of HF by Chinese medicine, and broadens new approaches for the treatment of HF. This study also has certain limitations. Since the process of myocardial fibrosis is the main mechanism of ventricular remodeling, this study did not investigate the effect of SQLXD on the formation of fibrosis. Also, we did not observe the effect of SQLXD on microcirculation in cardiac tissue. In the future study, we will further conduct some experiments to reveal the related mechanism.

\section{Acknowledgments}

Funding: Clinical Special Project Fund Project of Fujian University of Traditional Chinese Medicine (XB2018126).

\section{Footnote}

Reporting Checklist: The authors have completed the ARRIVE reporting checklist. Available at https://dx.doi. org/10.21037/atm-21-5350

Data Sharing Statement: Available at https://dx.doi. org/10.21037/atm-21-5350

Conflicts of Interest: All authors have completed the ICMJE uniform disclosure form (available at https://dx.doi. org/10.21037/atm-21-5350). The authors reported funding support from Clinical Special Project Fund Project of Fujian University of Traditional Chinese Medicine (XB2018126). The authors have no other conflicts of interest to declare.

Ethical Statement: The authors are accountable for all aspects of the work in ensuring that questions related 
to the accuracy or integrity of any part of the work are appropriately investigated and resolved. Experiments were performed under a project license (No. 909DWLL201901) granted by ethics committee of Xiamen University Affiliated Dongnan Hospital, in compliance with the National Laboratory Animal Management Regulations and guidelines for the care and use of animals.

Open Access Statement: This is an Open Access article distributed in accordance with the Creative Commons Attribution-NonCommercial-NoDerivs 4.0 International License (CC BY-NC-ND 4.0), which permits the noncommercial replication and distribution of the article with the strict proviso that no changes or edits are made and the original work is properly cited (including links to both the formal publication through the relevant DOI and the license). See: https://creativecommons.org/licenses/by-nc-nd/4.0/.

\section{References}

1. Ambrosy AP, Fonarow GC, Butler J, et al. The global health and economic burden of hospitalizations for heart failure: lessons learned from hospitalized heart failure registries. J Am Coll Cardiol 2014;63:1123-33.

2. McDonagh TA, Metra M, Adamo M, et al. 2021 ESC Guidelines for the diagnosis and treatment of acute and chronic heart failure. Eur Heart J 2021;42:3599-26. Erratum in: Eur Heart J 2021. [Epub ahead of print]. doi: 10.1093/eurheartj/ehab670.

3. Mao JY, Zhu MJ. Expert consensus on diagnosis and treatment of chronic heart failure in traditional Chinese medicine. Journal of Traditional Chinese Medicine 2014;55:1258-60.

4. Chen KJ, Wu ZG, Zhu MJ, et al. Expert consensus on the diagnosis and treatment of chronic heart failure with integrated traditional Chinese and western medicine. Chinese Journal of Integrated Traditional and Western Medicine 2016;36:133-41.

5. Sun XP, Liu L, Zou GL, et al. Shenqiyixin Decoction add-subtract in the treatment of chronic heart failure: clinical observation of 60 cases. Information on Traditional Chinese Medicine 2013;30:87-9.

6. Liu L, Gao J, Jin J. Clinical experience of professor LIU Li in the treatment of chronic heart failure with Shenqi Yixin Tang. Clinical Journal of Traditional Chinese Medicine 2015;27:1243-5.

7. Jin J, Cui L, Zou GL, et al. Effects of Shenqi Yixin Fang on Myocardial Structure and Cytokines in Doxorubicin- induced Chronic Heart Failure Rats. Chinese Journal of Experimental Traditional Medical Formulae 2014;20:128-32.

8. Wu L, Su Y, Tian ZK, et al. Effect of Shenqi Lixin Prescription on Hemodynamics, ANP, ALD and AngII in Heart Failure Rats. Acta Chinese Medicine and Pharmacology 2016;44:44-7.

9. Wu S, Zhu L, Yang J, et al. Hydrogen-containing saline attenuates doxorubicin-induced heart failure in rats. Pharmazie 2014;69:633-6.

10. Hayward R, Hydock DS. Doxorubicin cardiotoxicity in the rat: an in vivo characterization. J Am Assoc Lab Anim Sci 2007;46:20-32.

11. Kang PM, Izumo S. Apoptosis in heart: basic mechanisms and implications in cardiovascular diseases. Trends Mol Med 2003;9:177-82.

12. Rong X, Ge D, Yu L, et al. Enalapril attenuates endoplasmic reticulum stress and mitochondrial injury induced by myocardial infarction via activation of the TAK1/NFAT pathway in mice. Exp Ther Med 2020;19:972-80.

13. Osorio JC, Stanley WC, Linke A, et al. Impaired myocardial fatty acid oxidation and reduced protein expression of retinoid $X$ receptor-alpha in pacing-induced heart failure. Circulation 2002;106:606-12.

14. Liu C, Lin JD. PGC-1 coactivators in the control of energy metabolism. Acta Biochim Biophys Sin (Shanghai) 2011;43:248-57.

15. Ruiz-Velasco A, Liu W, Wang X. T4 ERK5 degradation: a turning point from compensated metabolic cardiomyopathy to heart failure. Heart 2018;104:A2.

16. Hauck L, Stanley-Hasnain S, Fung A, et al. Cardiacspecific ablation of the $\mathrm{E} 3$ ubiquitin ligase $\mathrm{Mdm} 2$ leads to oxidative stress, broad mitochondrial deficiency and early death. PLoS One 2017;12:e0189861.

17. Ashok D, Sidor A, O'Rourke B. Enhancing mitochondrial biogenesis with a CRISPR/ndCas9 adenoviral vector system in cardiomyocytes. Biophys J 2018;114:662A.

18. Qin Q, Lin N, Huang H, et al. Ginsenoside Rg1 ameliorates cardiac oxidative stress and inflammation in streptozotocin-induced diabetic rats. Diabetes Metab Syndr Obes 2019;12:1091-103.

19. Chen W, Sun Q, Ju J, et al. Effect of Astragalus Polysaccharides on Cardiac Dysfunction in $\mathrm{db} / \mathrm{db}$ Mice with Respect to Oxidant Stress. Biomed Res Int 2018;2018:8359013.

20. Lee YS, Kang YJ, Kim HJ, et al. Higenamine reduces apoptotic cell death by induction of heme oxygenase-1 
in rat myocardial ischemia-reperfusion injury. Apoptosis 2006;11:1091-100.

21. Chen C, Cui J, Lu H, et al. Modeling of the role of a Baxactivation switch in the mitochondrial apoptosis decision. Biophys J 2007;92:4304-15.

22. Youle RJ, Strasser A. The BCL-2 protein family: opposing activities that mediate cell death. Nat Rev Mol Cell Biol 2008;9:47-59.

23. Gogiraju R, Xu X, Bochenek ML, et al. Endothelial p53 deletion improves angiogenesis and prevents cardiac fibrosis and heart failure induced by pressure overload in mice. J Am Heart Assoc 2015;4:e01770.

24. Helton ES, Chen X. p53 modulation of the DNA damage response. J Cell Biochem 2007;100:883-96.

25. Xia P, Liu Y, Cheng Z. Signaling Pathways in Cardiac Myocyte Apoptosis. Biomed Res Int 2016;2016:9583268.

26. Zhang Y, Köhler K, Xu J, et al. Inhibition of p53 after acute myocardial infarction: reduction of apoptosis is counteracted by disturbed scar formation and cardiac rupture. J Mol Cell Cardiol 2011;50:471-8.

27. Hoshino A, Mita Y, Okawa Y, et al. Cytosolic p53 inhibits Parkin-mediated mitophagy and promotes mitochondrial dysfunction in the mouse heart. Nat Commun
2013;4:2308.

28. Huang Q, Gao S, Zhao D, et al. Review of ginsenosides targeting mitochondrial function to treat multiple disorders: current status and perspectives. J Ginseng Res. 2021;45:371-9.

29. Liu J, Li Y, Bian X, et al. Astragaloside IV alleviates heart failure by regulating SUMO-specific protease 1. Exp Ther Med 2021;22:1076.

30. Zhang Z, Wang J, Zhu Y, et al. Astragaloside IV alleviates myocardial damage induced by type 2 diabetes via improving energy metabolism. Mol Med Rep 2019;20:4612-22.

31. Jiang FL, Leo S, Wang XG, et al. Effect of tanshinone IIA on cardiomyocyte hypertrophy and apoptosis in spontaneously hypertensive rats. Exp Ther Med 2013;6:1517-21.

32. Zhou H, Yuan Y, Liu Y, et al. Icariin attenuates angiotensin II-induced hypertrophy and apoptosis in H9c2 cardiomyocytes by inhibiting reactive oxygen species-dependent JNK and p38 pathways. Exp Ther Med 2014;7:1116-22.

(English Language Editor: J. Chapnick)
Cite this article as: Zhuang J, Zhu J, Dou Y, Chen X, Chen H, Liu X, Lin G, Ruan F. Shenqi Lixin Decoction improves cardiac function in rats with adriamycin-induced heart failure through modulation of PGC- $1 \alpha$ and mitochondrial apoptosis pathway. Ann Transl Med 2021;9(20):1592. doi: 10.21037/atm-21-5350 